Research Article

\title{
Adaptive Dual-Polarization Filtering Method for Countering Active Jamming
}

\author{
Qiang Zhang $\mathbb{D}^{\text {, }}$, Rui Zhou $\mathbb{D}^{\mathbb{D}}$, Xianbo Shi $\mathbb{D}$, and Yixin Zhao \\ College of Air Traffic Management, Civil Aviation Flight University of China, Guanghan 618307, China \\ Correspondence should be addressed to Rui Zhou; zhouruihello@163.com
}

Received 12 January 2020; Revised 25 March 2020; Accepted 8 April 2020; Published 14 May 2020

Academic Editor: Giuseppe Castaldi

Copyright ( 2020 Qiang Zhang et al. This is an open access article distributed under the Creative Commons Attribution License, which permits unrestricted use, distribution, and reproduction in any medium, provided the original work is properly cited.

In order to counter active jamming, an adaptive polarization filtering method based on dual polarization radar is put forward. First, the signal flow principle of the dual polarization radar and its signal model are introduced. Then, the weighted coefficient matrices of the polarization filter are calculated adaptively according to the actual work situation of the current radar. Finally, the specific polarization filtering algorithm and the output criterion of the optimal filtering results are given. Experimental results show that this method does not need to know the type, quantity, combination mode, polarization characteristics, and other prior knowledge of active jamming but has well effect on both active deception jamming and active blanket jamming, so it has strong engineering application value.

\section{Introduction}

Active jamming is the main form of electromagnetic interference for radar systems, which can actively transmit interference electromagnetic wave and make the target echo signal received by the radar mix with a lot of active jamming signal, so as to achieve the aim of interfering normal target search, detection, and tracking [1-3]. The condition of radar interfered by active jamming is shown in Figure 1. Here, a target is just in the radar beam, so the target will certainly reflect the radar electromagnetic wave, while the active jamming in the electronic countermeasure airplane is transmitting interference electromagnetic wave to the radar; hence, it will make the radar at the same time receive the signals including both the target echo and the transmitting electromagnetic wave from active jamming and further affect the target search, detection, and tracking.

Active jamming includes active deception jamming and active blanket jamming. Especially, active deception jamming contains range deception jamming (RDJ), angle deception jamming (ADJ), frequency-shifting jamming
(FSJ), interrupted sampling repeater jamming (ISRJ), cross-polarization jamming (CPJ), active echo cancellation (AEC), and so on; active blanket jamming contains blinking noise jamming (BNJ), polarization agile jamming (PAJ), and so on [4-7]. Above all, there are more and more types of jamming in electronic warfare, so no method can counter all the types of jamming. But now, polarization technology is paid more attention for countering active jamming, because it has better effectiveness than frequency, time, and space algorithms. For example, polarization technologies are perfectly used to counter RDJ [8], ISRJ [9, 10], ADJ [11], CPJ [7], PAJ [12], and BNJ [13]. Nevertheless, most of the above techniques are just, respectively, for a certain type of active jamming. So, an adaptive filtering method for countering active jamming based on the dual polarization radar is proposed in this paper, which does not rely on any prior information of the jamming but is able to counter most types of the active jamming, such as RDJ, FSJ, BNJ, PAJ, and CPJ.

The remainder of this paper is organized as follows. The principle of the dual polarization radar and its signal 


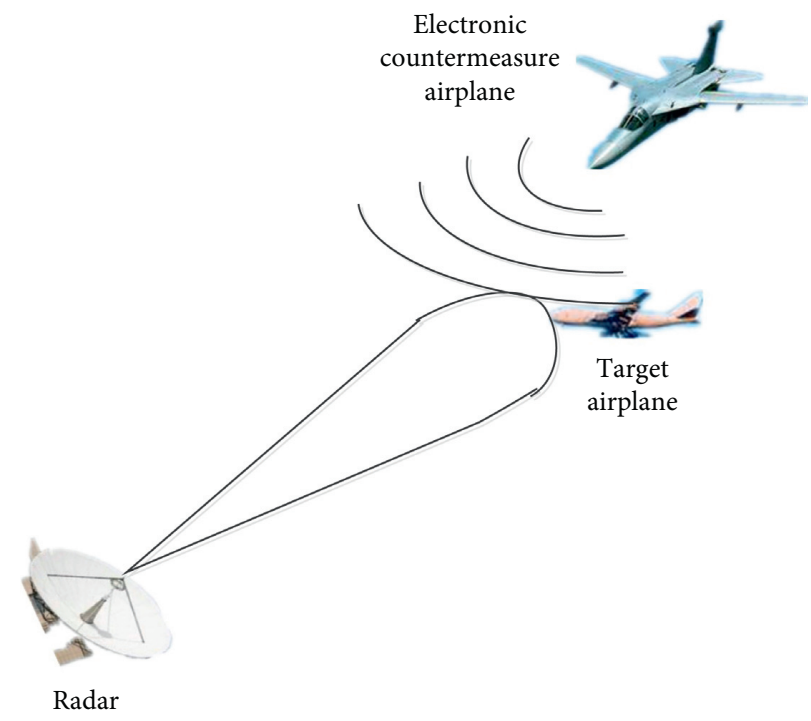

FIgURE 1: Radar interfered by active jamming.

model while countering active jamming are introduced in Section 2. Then, Section 3 addresses the specific algorithm and realization steps of the adaptive filtering method for countering active jamming. Experimental results and analysis are shown in Section 4. Finally, Section 5 concludes the paper.

\section{Principle of Dual Polarization Radar}

The principle diagram of the dual polarization radar system is shown in Figure 2, which mainly includes dual polarization antenna, transceiver front-end, frequency synthesizer/intermediate frequency/video frequency unit, signal processing unit, and information processing unit. The function of the dual polarization antenna is to radiate electromagnetic waves into the air and to collect electromagnetic waves from the air; the transceiver front-end is composed of the transmitter and the receiver, and it can also realize the switch control function of the transceiver; the frequency synthesizer/intermediate frequency/video frequency unit can complete the up and down conversion of frequency of radar signal; the signal processing unit contains IF (intermediate frequency) AD (analog-digital) conversion, signal accumulation, pulse compression, and other functions; the information processing unit primarily performs the functions of target detection, recognition, and tracking.

Dual polarization radar is a kind of radar system which can transmit electromagnetic waves with vertical polarization but receive those with vertical polarization and horizontal polarization at the same time. It can obtain echo signals of sum, azimuth difference, and elevation difference via vertical and horizontal polarization channels, respectively. Therefore, six channel signals can be acquired at the time of $t_{n}$, and they can be expressed as a matrix of $x\left(t_{n}\right), x\left(t_{n}\right)$ is shown in equation (1), including $x_{1}\left(t_{n}\right), x_{2}\left(t_{n}\right), x_{3}\left(t_{n}\right), x_{4}\left(t_{n}\right), x_{5}\left(t_{n}\right)$, and $x_{6}\left(t_{n}\right)$, in which they express sum with vertical polarization, sum with horizontal polarization, azimuth difference with vertical polarization, azimuth difference with horizontal polarization, elevation difference with vertical polarization, and elevation difference with horizontal polarization, respectively:

$$
x\left(t_{n}\right)=\left[\begin{array}{l}
x_{1}\left(t_{n}\right) \\
x_{2}\left(t_{n}\right) \\
x_{3}\left(t_{n}\right) \\
x_{4}\left(t_{n}\right) \\
x_{5}\left(t_{n}\right) \\
x_{6}\left(t_{n}\right)
\end{array}\right] .
$$

In the case of active jamming, the electromagnetic wave signals received by the dual polarization radar include both the reflected signal from the target and the transmitted signal by the active jamming. As a result, the signals received by the six channels can be described as follows [14]:

$$
\begin{aligned}
& x_{1}\left(t_{n}\right)=S\left(t_{n}\right)+\sum J_{i}\left(t_{n}\right)+N_{1}\left(t_{n}\right), \\
& x_{2}\left(t_{n}\right)=p_{I} S\left(t_{n}\right)+\sum a_{i} J_{i}\left(t_{n}\right)+N_{2}\left(t_{n}\right), \\
& x_{3}\left(t_{n}\right)=D_{A}\left(t_{n}\right)+\sum b_{i} J_{i}\left(t_{n}\right)+N_{3}\left(t_{n}\right), \\
& x_{4}\left(t_{n}\right)=f D_{A}\left(t_{n}\right)+\sum c_{i} J_{i}\left(t_{n}\right)+N_{4}\left(t_{n}\right), \\
& x_{5}\left(t_{n}\right)=D_{E}\left(t_{n}\right)+\sum d_{i} J_{i}\left(t_{n}\right)+N_{5}\left(t_{n}\right), \\
& x_{6}\left(t_{n}\right)=g D_{E}\left(t_{n}\right)+\sum e_{i} J_{i}\left(t_{n}\right)+N_{6}\left(t_{n}\right) .
\end{aligned}
$$




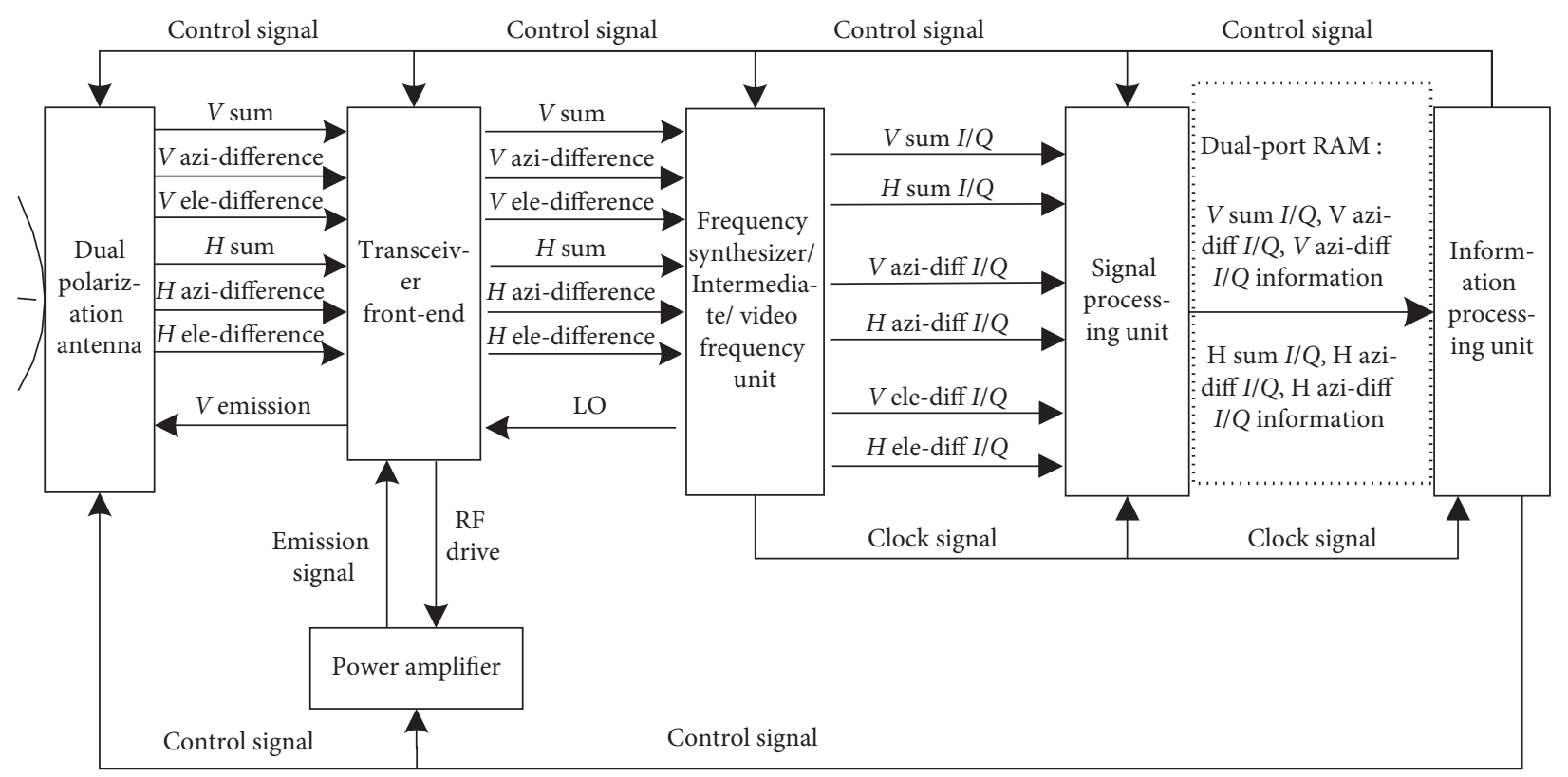

Figure 2: Principle diagram of dual polarization radar.

Here, $S\left(t_{n}\right)$ is the reflected echo signal of the target obtained by the vertical polarization sum channel; $J_{i}\left(t_{n}\right)$ represents the interference signal from the $i$ th active jamming obtained by the vertical polarization sum channel; $N_{1}\left(t_{n}\right), N_{2}\left(t_{n}\right), N_{3}\left(t_{n}\right), N_{4}\left(t_{n}\right), N_{5}\left(t_{n}\right)$, and $N_{6}\left(t_{n}\right)$ are the noise signals of the related channel respectively; $p_{I}$ is the cross-polarization ratio of the target; $a_{i}, b_{i}, c_{i}, d_{i}$, and $e_{i}$ are the receiving coefficients of the related channel to the vertical polarization sum channel for the transmitting electromagnetic wave signal of the $i$ th active jamming; $D_{A}\left(t_{n}\right)$ and $D_{E}\left(t_{n}\right)$ are the reflected echo signals of the target obtained by the vertical polarization azimuth difference channel and the vertical polarization elevation difference channel, respectively; and $f$ and $g$ are the receiving coefficients of the horizontal polarization azimuth difference channel and the horizontal polarization elevation difference channel to the related channel for the reflected echo signal.

\section{Adaptive Filtering Method}

The adaptive dual-polarization filtering method is shown in Figure 3, and it can be realized through the following six steps:

(1) At the time of $t_{n-N}, \ldots, t_{n-1}, t_{n}, t_{n+1}, \ldots, t_{n+N}$, the dual polarization radar can, respectively, obtain $x\left(t_{n-N}\right), \ldots, x\left(t_{n-1}\right), x\left(t_{n}\right), x\left(t_{n+1}\right), \ldots, x\left(t_{n+N}\right)$, which are the electromagnetic wave signals of the six channels. And those are the input of the adaptive dual polarization filtering method.

(2) Compute $R_{x E}$; it is the average value of the autocorrelation coefficients of the received signal matrices from the time of $t_{n-N}$ to $t_{n-1}$. Similarly, compute $R_{x x 1 E}$; it is the average value of the cross- correlation coefficients of the received signal matrices and the vertical polarization sum signal from the time of $t_{n-N}$ to $t_{n-1}$. The equations are expressed as (3) and (4), where superscript $\mathrm{T}$ represents the transpose of the matrix and superscript ${ }^{*}$ represents the adjoint of the matrix:

$$
\begin{gathered}
R_{x E}=\frac{1}{N} \sum_{m=1}^{N} x\left(t_{n-m}\right) x^{\mathrm{T}}\left(t_{n-m}\right), \\
R_{x x 1 E}=\frac{1}{N} \sum_{m=1}^{N} x\left(t_{n-m}\right) x_{1}^{*}\left(t_{n-m}\right) .
\end{gathered}
$$

(3) The same procedure is performed as given in Step (2); compute $R_{x L}$ and $R_{x x 1 L}$ through equations (5) and (6). $R_{x L}$ is the average value of the autocorrelation coefficients of the received signal matrices from the time of $t_{n+1}$ to $t_{n+N} . R_{x x 1 L}$ is the average value of the cross-correlation coefficients of the received signal matrices and the vertical polarization sum signal from the time of $t_{n+1}$ to $t_{n+N}$ :

$$
\begin{gathered}
R_{x L}=\frac{1}{N} \sum_{m=1}^{N} x\left(t_{n+m}\right) x^{\mathrm{T}}\left(t_{n+m}\right), \\
R_{x x 1 L}=\frac{1}{N} \sum_{m=1}^{N} x\left(t_{n+m}\right) x_{1}^{*}\left(t_{n+m}\right) .
\end{gathered}
$$

(4) Calculate the weighted coefficient matrices of the polarization filter. Set $H=\left[\begin{array}{llllll}1 & -1 & -1 & -1 & -1 & -1\end{array}\right]^{T}$, so the weighted coefficient matrices of $\widehat{W}_{E}, \widehat{W}_{L}$, and $\widehat{W}_{0}$ can be represented as follows: 
$W_{E}=R_{x E}^{-1} R_{x x 1 E}$,

$W_{L}=R_{x L}^{-1} R_{x x 1 L}$,

$W_{E}=\left[\begin{array}{l}W_{1 E} \\ W_{2 E} \\ W_{3 E} \\ W_{4 E} \\ W_{5 E} \\ W_{6 E}\end{array}\right]=\left[\begin{array}{c}1 \\ W_{2 E} \\ W_{3 E} \\ W_{4 E} \\ W_{5 E} \\ W_{6 E}\end{array}\right]$,

$W_{L}=\left[\begin{array}{l}W_{1 L} \\ W_{2 L} \\ W_{3 L} \\ W_{4 L} \\ W_{5 L} \\ W_{6 L}\end{array}\right]=\left[\begin{array}{c}1 \\ W_{2 L} \\ W_{3 L} \\ W_{4 L} \\ W_{5 L} \\ W_{6 L}\end{array}\right]$,

$W_{0}=\left[\begin{array}{l}W_{10} \\ W_{20} \\ W_{30} \\ W_{40} \\ W_{50} \\ W_{60}\end{array}\right]=\frac{1}{2}\left(W_{E}+W_{L}\right)=\left[\begin{array}{c}1 \\ W_{20} \\ W_{30} \\ W_{40} \\ W_{50} \\ W_{60}\end{array}\right]$,

$\widehat{W}_{E}=W_{E} \cdot H=\left[\begin{array}{c}1 \\ -W_{2 E} \\ -W_{3 E} \\ -W_{4 E} \\ -W_{5 E} \\ -W_{6 E}\end{array}\right]$,

$\widehat{W}_{L}=W_{L} \cdot H=\left[\begin{array}{c}1 \\ -W_{2 L} \\ -W_{3 L} \\ -W_{4 L} \\ -W_{5 L} \\ -W_{6 L}\end{array}\right]$,

$\widehat{W}_{0}=W_{0} \cdot H=\left[\begin{array}{c}1 \\ -W_{20} \\ -W_{30} \\ -W_{40} \\ -W_{50} \\ -W_{60}\end{array}\right]$.
(5) Polarization filtering process is carried out on the received signals according to equation (8), so three filtering results can be obtained:

$$
\left[\begin{array}{l}
y_{E}\left(t_{n}\right) \\
y_{L}\left(t_{n}\right) \\
y_{0}\left(t_{n}\right)
\end{array}\right]=\left[\begin{array}{c}
x^{T}\left(t_{n}\right) \\
x^{T}\left(t_{n}\right) \\
x^{T}\left(t_{n}\right)
\end{array}\right]\left[\begin{array}{lll}
\widehat{W}_{E} & \widehat{W}_{L} & \widehat{W}_{0}
\end{array}\right]=\left[\begin{array}{c}
x^{T}\left(t_{n}\right) \widehat{W}_{E} \\
x^{T}\left(t_{n}\right) \widehat{W}_{L} \\
x^{T}\left(t_{n}\right) \widehat{W}_{0}
\end{array}\right] .
$$

(6) Set $\quad P_{E}\left(t_{n}\right)=\left|y_{E}\left(t_{n}\right)\right|^{2}, \quad P_{L}\left(t_{n}\right)=\left|y_{L}\left(t_{n}\right)\right|^{2}$, and $\varepsilon=0.09$; the optimal filtering result can be selected based on equation (9), which can be used for radar target detection and tracking with active jamming conditions:

$$
y\left(t_{n}\right)= \begin{cases}y_{E}\left(t_{n}\right) & \frac{P_{E}\left(t_{n}\right)}{P_{L}\left(t_{n}\right)}<\varepsilon, \\ y_{L}\left(t_{n}\right) & \frac{P_{L}\left(t_{n}\right)}{P_{E}\left(t_{n}\right)}<\varepsilon, \\ y_{0}\left(t_{n}\right) & \text { else. }\end{cases}
$$

\section{Experimental Results}

In order to verify the effectiveness of the adaptive filtering method based on the dual-polarization radar proposed in this paper, the actual collected data are used to the experimental analysis in this section. Peculiarly, the dual-polarization radar works in millimeter-wave band, with chirp signal, and its time duration-bandwidth product is 72 . In the experiment, we use RDJ with circle polarization to represent active deception jamming and use BNJ with circle polarization to represent active blanket jamming.

So, the experimental results are given as follows. Figure 4 shows the echo signal received by the vertical polarization sum channel of the dual polarization radar under the condition of active deception jamming. Figure 5 shows the echo signal received by the horizontal polarization sum channel at the same time. Figure 6 shows the result of the adaptive dual polarization filtering method proposed in this paper. Figure 7 shows the echo signal received by the vertical polarization sum channel of the dual-polarization radar under the condition of active blanket jamming. Figure 8 shows the echo signal received by the horizontal polarization sum channel at the same time. Figure 9 shows the result of the adaptive dual-polarization filtering method proposed in this paper. It can be found that the jamming is well suppressed, while the target echo signal has been retained effectively. Therefore, the proposed adaptive filtering method based on the dual polarization radar is extraordinarily effective against active jamming. 


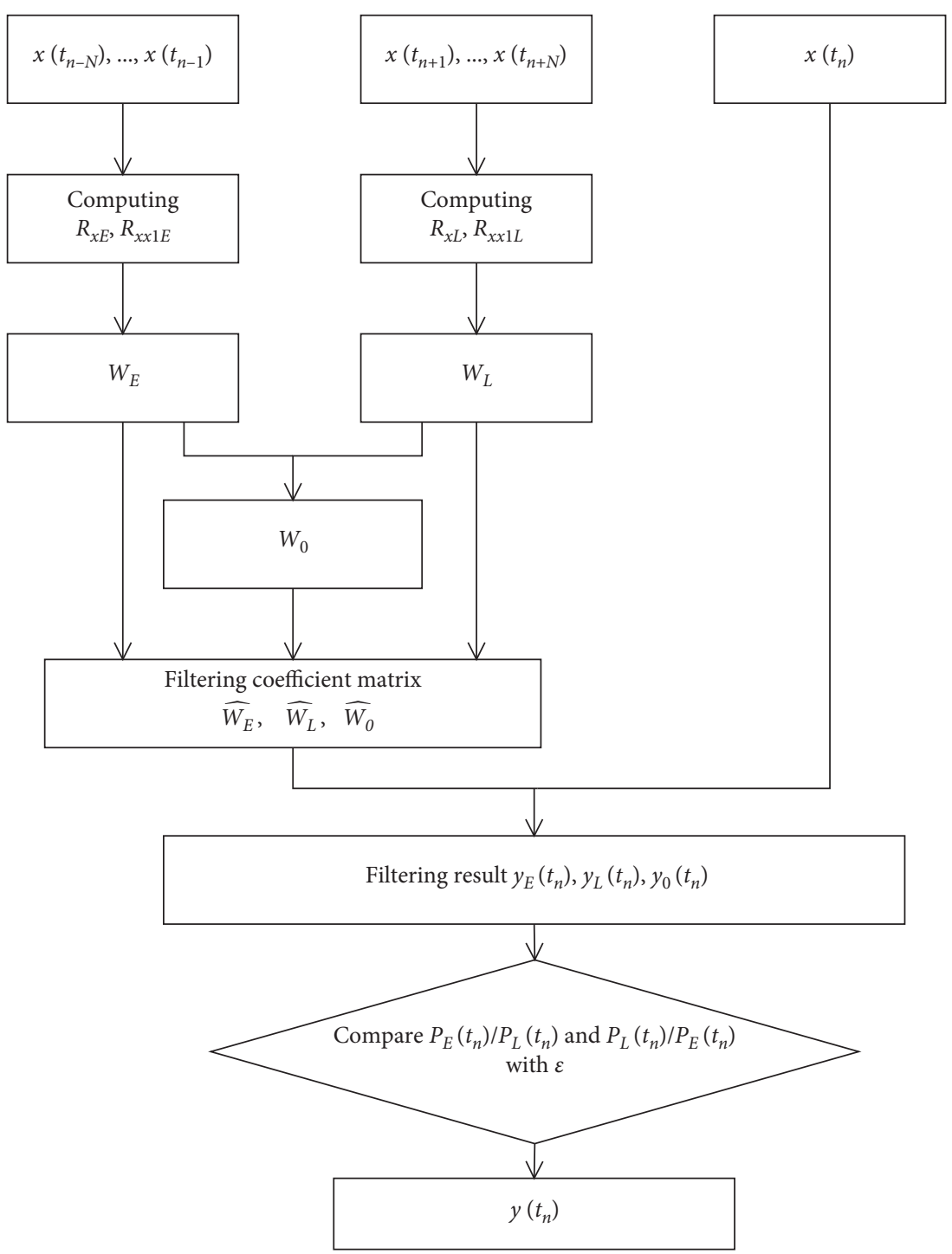

FIGURE 3: Flow chart of the adaptive filtering method.

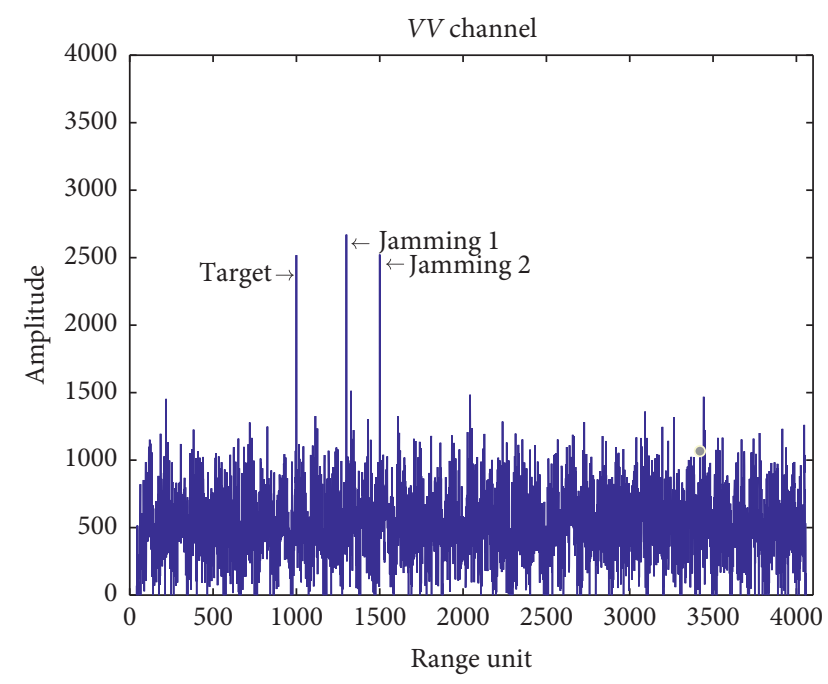

FIGURE 4: Echo signal received by the vertical polarization sum channel with active deception jamming.

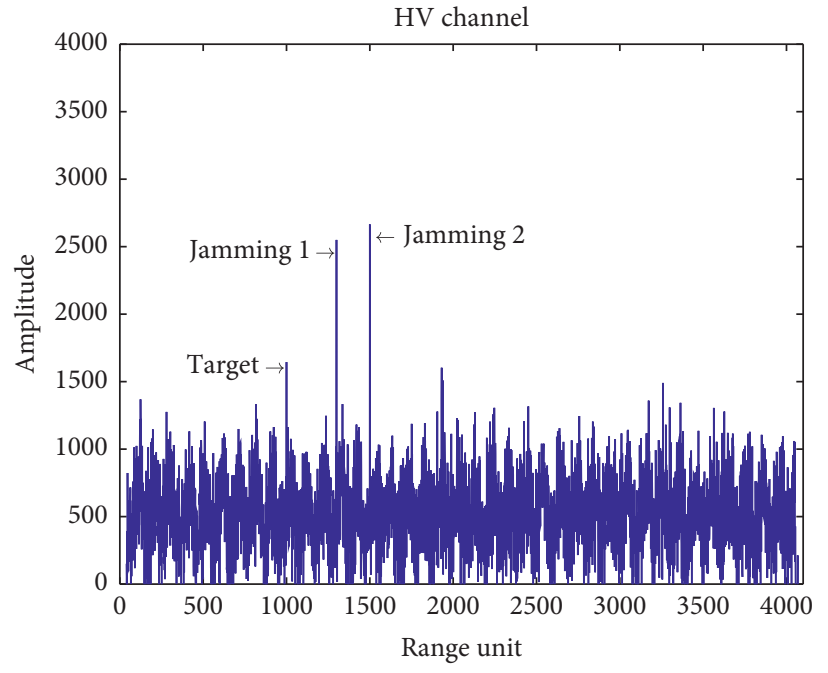

FIGURE 5: Echo signal received by the horizontal polarization sum channel with active deception jamming. 


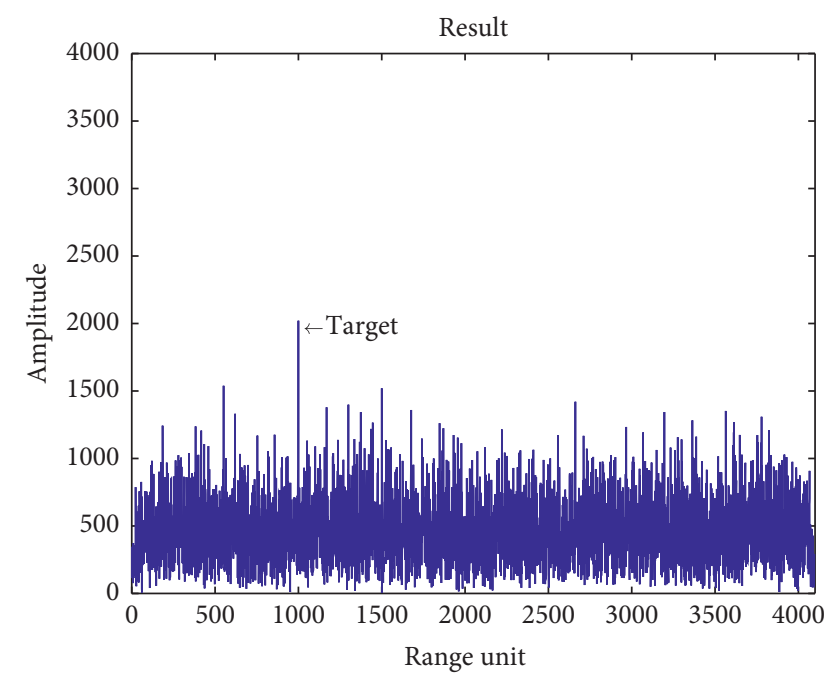

FIGURE 6: Result of adaptive dual-polarization filtering with active deception jamming.

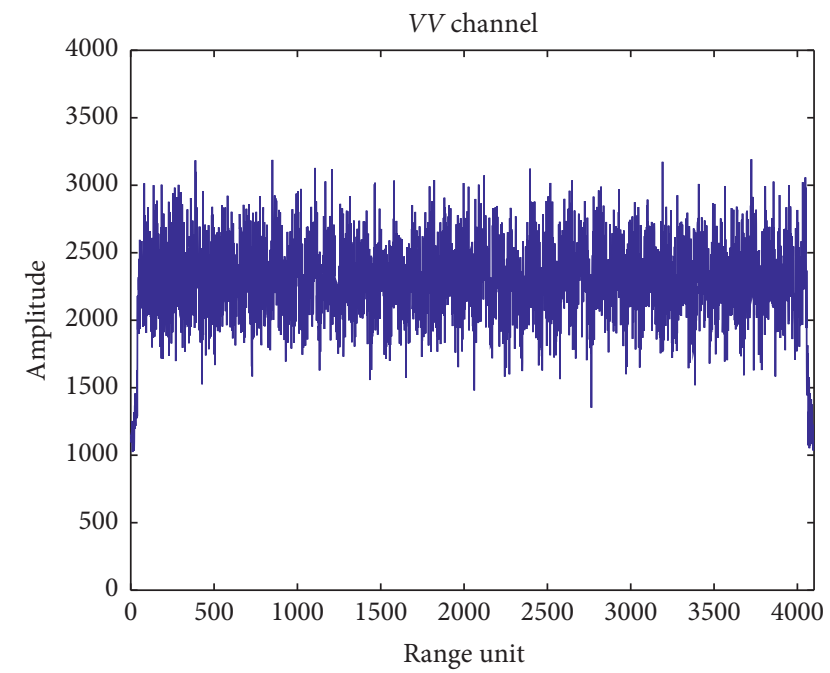

FIGURE 7: Echo signal received by vertical polarization sum channel with active blanket jamming.

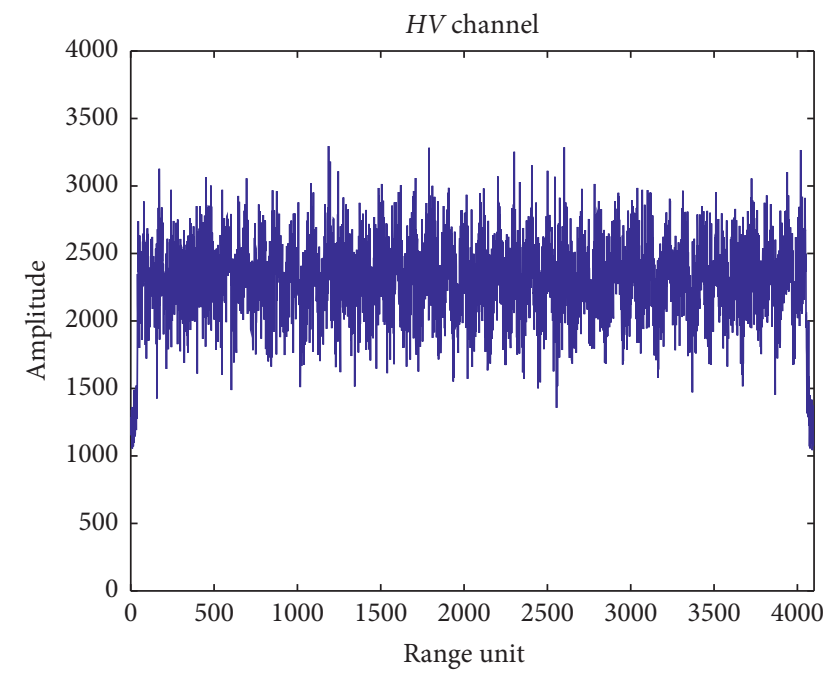

FIGURE 8: Echo signal received by horizontal polarization sum channel with active blanket jamming.

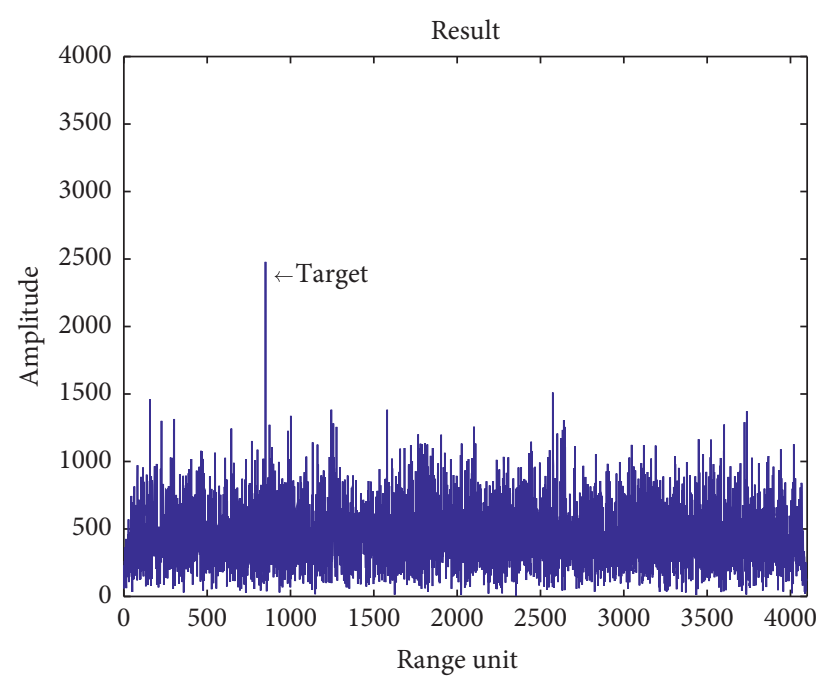

FIGURE 9: Result of adaptive dual polarization filtering with active blanket jamming.

\section{Conclusion}

An adaptive filtering method for countering active jamming based on the dual polarization radar is presented in the paper. It does not need to know the prior knowledge of type, quantity, combination mode, and polarization characteristics of active jamming, and the weighted coefficient matrices of the filtering method can be adaptively gained according to the actual scene; then, the best filtering result can be selected to use in target detection and tracking with condition of active jamming. In addition, the filtering method also has good universality and is effective for both active deception jamming and active blanket jamming in various scenarios. Significantly, it is suitable for monitoring radar, guidance radar, and fire control radar to improve detecting and tracking performance under active jamming conditions. Above all, the presented technique is robust and has strong engineering application value.

\section{Data Availability}

The data of radar echo used to support the findings of this study are available from the corresponding author upon request.

\section{Conflicts of Interest}

The authors declare that they have no conflicts of interest.

\section{Acknowledgments}

This work was supported by the National Key Research and Development Program of China (Grant no. 2016YFB0502403), Sichuan Science and Technology Program (Grant no. 2018GZ0167), the Safety Capacity Building Program of Civil Aviation Administration of China (Grant no. 0241928), and the Scientific Research Fund of Civil Aviation Flight University of China (Grant no. CJ2018-02). 


\section{References}

[1] H. Dai, X. Wang, and Y. Li, "Novel discrimination method of digital deceptive jamming in mono-pulse radar," Journal of Systems Engineering and Electronics, vol. 22, no. 6, pp. 910916, 2011.

[2] X. Pan, D. Feng, Q. Fu, W. Wang, Y. Liu, and G. Wang, "On deception jamming for countering bistatic ISAR based on subNyquist sampling," IET Radar, Sonar \& Navigation, vol. 8, no. 3, pp. 173-179, 2014.

[3] J.-W. Tao, "Performance analysis for interference and noise canceller based on hypercomplex and spatio-temporalpolarisation processes," IET Radar, Sonar \& Navigation, vol. 7, no. 3, pp. 277-286, 2013.

[4] J.-S. Lee and E. Pottie, Polarimetric Radar Imaging: From Basics to Applications, CRC Press, Boca Raton, FL, USA, 2008.

[5] Z. Shenghua, X. Dazhuan, J. Xueming, and H. Hua, "A study on active jamming to synthetic aperture radar," in Proceedings of the ICCEA 2004. 2004 3rd International Conference on Computational Electromagnetics and Its Applications, 2004, Beijing, China, November 2004.

[6] X. Tian and T. Bin, "Active deception jamming recognition of radar based on normalized wavelet decomposition power ratio," Journal of Data Acquisition \& Processing, vol. 28, no. 4, pp. 416-420, 2013.

[7] Y. Li, Radar Polarization Anti-Jamming Technology, National Defense Industry Press, Changsha, China, 2013.

[8] Y. Liu, Y. Z. Li, X. S. Wang, and S.-P. Xiao, "Discrimination of HRRP deception jamming based on polarization diversity receiving," Journal of System Engineering and Electronics, vol. 6, no. 10, 2011.

[9] Q. Zhang and W. Pan, "Countering method for active jamming based on dual-polarization radar seeker," International Journal of Microwave and Wireless Technologies, vol. 9, no. 5, pp. 1067-1073, 2016.

[10] D. Huan-Yao, L. Yong-Zhen, L. Yong, C. Yu-Liang, W. XueSong, and L. Mian-Quan, "Study on polarimetric measurement and radar target identification in instantaneous polarization radar system," in Proceedings of the IET International Radar Conference 2009, Guilin, China, April 2009.

[11] Q. Zhang and W. Pan, "Adaptive countering technique for angle deception based on dual polarization radar seeker," International Journal of Antennas and Propagation, vol. 2017, Article ID 2345491, 8 pages, 2017.

[12] Q. Xiaolin, J. Tao, Q. Xiaohui, Z. Min, Y. Shuqing, and Z. Qunxing, "Anti-millimeter wave polarization agile active jamming," in Proceedimgs of the 2007 International Conference on Microwave and Millimeter Wave Technology, Builin, China, April 2007.

[13] H. Dai, X. Wang, Y. Li, Y. Liu, and S. Xiao, "Main-lobe jamming suppression method of using spatial polarization characteristics of antennas," IEEE Transactions on Aerospace and Electronic Systems, vol. 48, no. 3, pp. 2167-2179, 2012.

[14] B. Varga and G. DeWolf, "Polarization ECCM technique for missile radar systems," US Patent US5311192, 1994. 